\title{
Canadian Experience with the Pipeline Embolization Device for Repair of Unruptured Intracranial Aneurysms
}

\author{
C.J. O’Kelly, J. Spears, M. Chow, J. Wong, M. Boulton, A. Weill, R.A. Willinsky, M. Kelly, and T.R. Marotta
}

\begin{abstract}
BACKGROUND AND PURPOSE: Flow-diverting stents, such as the PED, have emerged as a novel means of treating complex intracranial aneurysms. This retrospective analysis of the initial Canadian experience provides insight into technical challenges, clinical and radiographic outcomes, and complication rates after the use of flow-diverting stents for unruptured aneurysms.
\end{abstract}

MATERIALS AND METHODS: Cases were compiled from 7 Canadian centers between July 2008 and December 2010. Each center prospectively tracked their initial experience; these data were retrospectively updated and pooled for analysis.

RESULTS: During the defined study period, 97 cases of unruptured aneurysm were treated with the PED, with successful stent deployment in 94 cases. The overall complete or near-complete occlusion rate was 83\%, with a median follow-up at 1.25 years (range $0.25-2.5$ years). Progressive occlusion was witnessed over time, with complete or near-complete occlusion in $65 \%$ of aneurysms followed through 6 months, and $90 \%$ of aneurysms followed through 1 year. Multivariate analysis found previous aneurysm treatment and female sex predictive of persistent aneurysm filling. Most patients were stable or improved (88\%), with the most favorable outcomes observed in patients with cavernous carotid aneurysms. The overall mortality rate was $6 \%$. Postprocedural aneurysm hemorrhage occurred in 3 patients (3\%), while ipsilateral distal territory hemorrhage was observed in 4 patients (3.4\%).

CONCLUSIONS: Flow-diverting stents represent an important tool in the treatment of complex intracranial aneurysms. The relative efficacy and morbidity of this treatment must be considered in the context of available alternate interventions.

ABBREVIATIONS: $\mathrm{ICH}=$ intracerebral hemorrhage; $\mathrm{PED}=$ Pipeline embolization device

$F$ ow-diverting stents have emerged as a treatment option for complex intracranial aneurysms. These flexible, low-porosity stents limit flow into and out of intracranial aneurysms, resulting in thrombosis of the aneurysms over time. ${ }^{1}$ Early experience with these devices has suggested a high rate of aneurysm closure with

Received March 19, 2012; accepted after revision May 21.

From the Division of Neurosurgery (C.J.O., M.C.), Department of Surgery, University of Alberta, Edmonton, Alberta, Canada; Division of Neurosurgery, Department of Surgery (J.S.), and Department of Radiology (R.A.W., T.R.M.), University of Toronto, Toronto, Ontario, Canada; Division of Neurosurgery (J.W.), Department of Clinical Neurosciences, University of Calgary, Calgary, Alberta, Canada; Division of Neurosurgery (M.B.), Department of Surgery, University of Western Ontario, London, Ontario, Canada; Department of Radiology (A.W.), University of Montreal, Montreal, Quebec, Canada; and Division of Neurosurgery, Department of Surgery (M.K.), University of Saskatchewan, Saskatoon, Saskatchewan, Canada.

Previously presented in abstract form at: Canadian Congress of Neurological Sciences, Vancouver, British Columbia, Canada, June 17, 2011; World Fedaration of Interventional and Therapeutic Neuroradiology, Capetown, South Africa, November 9, 2011.

Please address correspondence to Dr. Cian J. O'Kelly, 2D1.02 Mackenzie Centre, 8440-112 St, Edmonton, Alberta, Canada, T6G 2B7; e-mail: cian.okelly@albertahealthservices.ca

三 Indicates article with supplemental on-line table.

http://dx.doi.org/10.3174/ajnr.A3224 limited morbidity. ${ }^{2,3}$ Flow-diverting stents have been used to successfully treat complex aneurysms with limited therapeutic alternatives, yet their role in general aneurysm management remains to be defined. Rare but significant complications, such as delayed aneurysm rupture and distal $\mathrm{ICH}$, have tempered enthusiasm for widespread use. Questions remain regarding the overall efficacy of the devices, the morbidity of treatment, long-term outcomes, and the cost-effectiveness of these expensive devices.

We present the initial Canadian experience with 1 flow-diverting stent, the PED (ev3; Plymouth, Minnesota). This retrospective analysis provides a real-world sense of the efficacy, limitations, and complications associated with the PED. In many cases the results are positive, but important complications require circumspect incorporation of these devices into cerebrovascular practice.

\section{MATERIALS AND METHODS}

This is a retrospective cohort study of all unruptured aneurysm cases treated with the PED in Canada between July 2008 and December 2010. Case data were prospectively gathered at each of the 7 participating centers. Local research ethics board approval was 
obtained at the University of Alberta. For the purpose of this study, the data were anonymized and pooled for retrospective analysis. During the initial portion of this study, the PED was only available through a Health Canada compassionate-use program. The initial cases at each site were proctored by expert users. The PED was considered primarily for cases where the alternativeeither surgery or conventional coiling-was felt to carry either excessive procedural risk or a high likelihood of failure to durably occlude the aneurysm. The PED was also considered as an alternative to vessel sacrifice for the treatment of large, complex anterior circulation aneurysms.

Stent placement and periprocedural management were not standardized but were generally uniform as a result of the guidance of a relatively small core group of proctors. Patients were preloaded with dual antiplatelet medication, mainly acetylsalicylic acid and clopidogrel, continued for an additional 6 months. Dual antiplatelet treatment was extended in cases of in-stent stenosis. Where possible, stents were deployed by using a tri-axial catheter system consisting of a larger shuttle-type guide catheter (Cook Vascular, Vandergrift, Pennsylvania), a flexible distal access guide catheter (Neuron; Penumbra, Alameda, California), and a microcatheter (Marksman, ev3, Irvine, California; or Renegade Hi-Flo, Boston Scientific, Natick, Massachusetts). Full heparinization was initiated before establishing the triaxial catheter system, aiming for 2-2.5 times the activated coagulation time. The number of stents deployed and the use of adjuvant coils was at the discretion of the treating physicians. Postdeployment angioplasty, generally using a $4 \times 10 \mathrm{~mm}$ to $4 \times 20 \mathrm{~mm}$ HyperGlide balloon (ev3), was occasionally required to address problems with stent kinking and inadequate vessel wall apposition.

Baseline characteristics were recorded, including patient demographics, mode of presentation, and aneurysm specifics such as location, largest diameter $(\mathrm{mm})$, neck width $(\mathrm{mm})$, and morphology. Periprocedural morbidity and mortality events were recorded, as were any significant clinical events through follow-up. The patients' clinical status was recorded at planned follow-up assessments. Standardized outcome assessments were not routinely performed at each institution and therefore could not be included in our analysis. Radiographic follow-up consisted of a combination of CTA, MRA, and DSA. Follow-up paradigms - in particular, technique and timing — varied between centers. Aneurysm closure was judged using all available radiographic modalities. Aneurysm filling was categorized as no significant change ( $95 \%-100 \%$ of baseline contrast filling), residual aneurysm (5\%95\% residual filling), near-complete occlusion (entry remnant, $<5 \%$ residual filling), and complete occlusion (no filling). Standardized assessment of contrast stasis was not available for analysis. In-stent stenosis was recorded for patients undergoing DSA, whereas the residual aneurysm mass was estimated from the cross-sectional imaging (CTA and MRA). Residual aneurysm mass was noted as increased, not significantly changed (75\%$100 \%$ of baseline), reduced ( $25 \%-75 \%$ of baseline), or significantly reduced ( $<25 \%$ of baseline).

All data were analyzed using SAS statistical software (version 9.2; SAS, Cary, North Carolina). Kaplan-Meier methodology was followed for generation of time-to-event curves. Univariate and multivariate analyses of outcomes were conducted by using logis-
Table 1: Clinical presentations

\begin{tabular}{lc}
\hline \multicolumn{1}{c}{ Clinical Presentation } & $\begin{array}{c}\text { Number of Patients } \\
\text { (\%) }\end{array}$ \\
\hline Visual complaints & $36(37.1 \%)$ \\
Diplopia (cranial nerve palsy) & $24(24.7 \%)$ \\
Visual loss & $12(12.4 \%)$ \\
Headaches & $14(14.4 \%)$ \\
Incidental & $25(25.8 \%)$ \\
Recanalization of previously treated aneurysm & $17(17.5 \%)$ \\
Neurological deficit & $4(4.12 \%)$ \\
Other & $1(1.0 \%)$ \\
\hline
\end{tabular}

tic regression or Cox proportional hazards modeling, where appropriate.

\section{RESULTS}

\section{Baseline Characteristics}

A total of 109 PED cases were performed at the participating centers between June 2008 and December 2010, including 97 patients with unruptured aneurysms (12 patients with ruptured aneurysms were excluded from this analysis). The mean age at the time of treatment was 56.5 years. Most patients were female $(82.5 \%)$. Visual complaints (diplopia, visual field changes, and visual acuity changes) were the most common mode of presentation (37.1\%); other presentations are summarized in Table 1. Patients presenting with other neurologic deficits were generally with mass effect, secondary to large partially thrombosed aneurysms, while 1 patient presented with emboli from a similar aneurysm. One patient (other) presented with middle ear effusion, secondary to a petrosal segment carotid artery aneurysm. ${ }^{4}$

Most aneurysms in this series were large and wide-neck aneurysms. The mean aneurysm diameter was $19 \mathrm{~mm}$ (range 2-60 $\mathrm{mm}$ ), while the mean neck width was $8 \mathrm{~mm}$ (range 2-25 mm). The average neck-to-dome ratio was 0.48 . Eight percent of the aneurysms had a fusiform morphology. Partial thrombosis of the aneurysm sac was observed in $25.7 \%$ of cases. A detailed summary of aneurysm location is shown in Fig 1. Aneurysms were predominantly located in the anterior circulation $(81.4 \%)$, with $34 \%$ of these in the cavernous segment and $58 \%$ in the supraclinoid segment.

\section{Procedural Specifics}

The PED was successfully deployed in 94 of 97 cases (96.9\%). In 2 cases, the PED could not be deployed due to an inability to gain stable catheter access across the neck of a giant aneurysm. In 1 of these cases, the parent vessel was ultimately sacrificed while conservative management was opted for in the other case. In the third case, the PED retracted into the aneurysm, necessitating parent vessel occlusion. The patient had previously passed a test occlusion and tolerated the permanent vessel occlusion.

Of cases where device deployment was achieved, the mean number of PEDs used was 1.8 (range 1-13). In over half of the cases $(53.2 \%$ ), a single PED was deployed, while 2 or 3 stents were used in $31.9 \%$ and $9.6 \%$ of cases, respectively. Five or more stents (range 5-13) were used in the remaining 5\% of cases. Multiple PEDs were used more frequently in fusiform aneurysms (mean 4.1 PEDs, $P<.001$ ). Additional coils were placed in the aneurysm sac in $5.1 \%$ of cases. In most cases, partial coiling of the aneurysm 


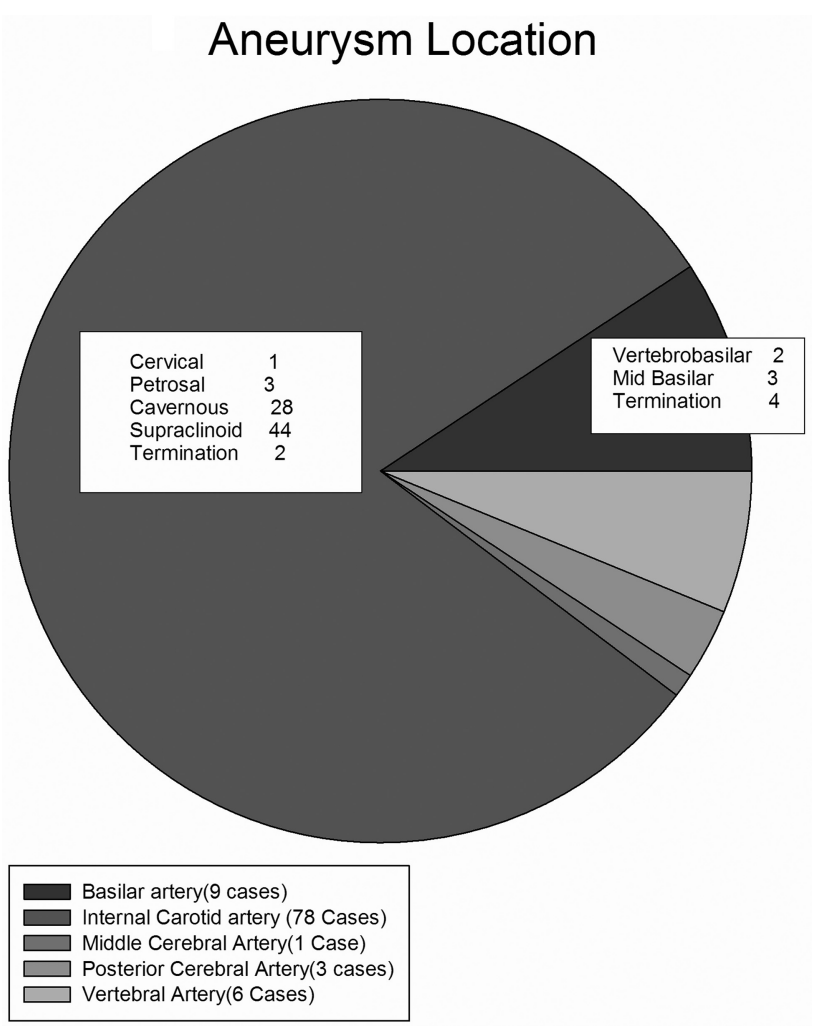

FIG 1. Aneurysm location for cases in the Canadian experience. Seventy-nine $(81.4 \%)$ cases were located in the anterior circulation, with $18(18.6 \%)$ located in the posterior circulation.

was undertaken to disrupt entry flow into the aneurysm sac. In 1 vertebrobasilar junction aneurysm, the nonstented vertebral artery was occluded with coils at its distal V4 segment. As expected, most aneurysms continued to fill on the postdeployment control angiogram, with only $2 \%$ of cases showing complete or near-complete closure at this stage.

\section{Radiographic Outcomes}

Median follow-up for the entire cohort was 1.25 years (range 0.25-2.5 years). At the most recent radiographic follow-up, $68.4 \%$ of aneurysms were completely occluded, with near-occlusion (entry remnant) noted in an additional $15.8 \%$ of aneurysms. As such, complete or near-complete occlusion was noted in $84.2 \%$ of cases. Reduced aneurysm filling (5\%-95\% residual) was observed in $4.2 \%$ of cases, while $11.6 \%$ of cases showed persistent filling ( $>95 \%$ residual). The progressive closure of aneurysms over time is demonstrated in Fig 2. Persistent filling of the aneurysm was observed infrequently beyond 1 year of follow-up. Predictors of aneurysm closure were assessed in a univariate proportional hazards model (Table 2). Recurrence of a previously treated aneurysm was associated with decreased aneurysm closure. Similarly, location in the posterior circulation demonstrated a trend for reduced aneurysm closure over time. Multivariate analysis was performed for statistically significant univariate predictors $(P<.10)$, with sex and previous treatment independently associated with reduced rates of aneurysm closure (Table 2).

Data on residual aneurysm mass were available for 82 of 94 cases $(87 \%)$. Overall, 31 cases (38\%) showed no change in
Table 2: Predictors of aneurysm closure over time: univariate survival analysis

\begin{tabular}{lcc}
\hline \multicolumn{1}{c}{ Covariate } & Hazard Ratio & $\boldsymbol{P}$ Value \\
\hline PED number & 1.05 & .36 \\
Age & 0.99 & .69 \\
Female gender & 0.52 & .03 \\
Coil use & 0.99 & .69 \\
Posterior circulation & 0.57 & .07 \\
Aneurysm diameter & 0.99 & .52 \\
Aneurysm neck & 1.01 & .55 \\
Associated thrombus & 1.05 & .82 \\
Recanalized & 0.36 & .002 \\
\hline
\end{tabular}

Note:-PED number refers to number of Pipeline stents used to treat the aneurysm. Coil use refers to the use of additional coils at the time of PED treatment. Recanalized refers to a recurrence of a previously treated aneurysm.

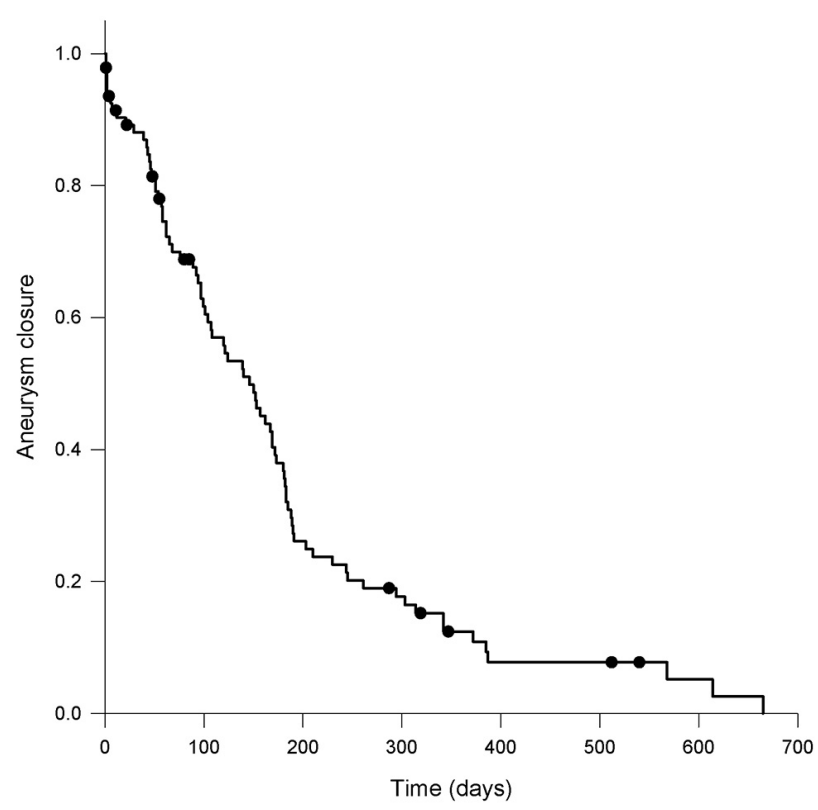

FIG 2. Kaplan-Meier survival plot demonstrating the rate of aneurysm closure over time. Shaded circles indicate censored observations.

residual aneurysm size ( $75 \%-100 \%$ of baseline). Reduced $(25 \%-$ $75 \%$ of baseline) or significantly reduced ( $<25 \%$ of baseline) size was observed in $27(33 \%)$ and $21(26 \%)$ cases, respectively. As indicated in Fig 2, the proportion of cases showing reduction in size increases through long-term follow-up. Aneurysm growth occurred in 3 cases and was associated with adverse neurologic outcomes (described below).

DSA follow-up was available for analysis of in-stent stenosis and vessel occlusion in 58 (61\%) cases. Of the patients undergoing angiography, most (90\%) showed no evidence of in-stent stenosis. There were 2 cases (3\%) of mild in-stent stenosis, which resolved spontaneously in subsequent follow-up. Two cases (3\%) demonstrated significant, but stable, stenosis of $50 \%$ and $70 \%$. There were 2 cases of delayed vessel occlusion (3\%) occurring 7 and 12 months after the stent placement procedure. Neither of these cases was associated with permanent neurologic deficit, though 1 patient had a small area of diffusion restriction noted on MR imaging. 
Table 3: Predictors of mortality: univariate logistic regression analysis

\begin{tabular}{lcc}
\hline \multicolumn{1}{c}{ Covariate } & Odds Ratio & $\boldsymbol{P}$ Value \\
\hline Age & 0.970 & .27 \\
Female gender & 5.77 & .04 \\
Mode of presentation & $\mathrm{N} / \mathrm{A}^{\mathrm{a}}$ & .99 \\
Posterior circulation & 10.57 & .01 \\
Aneurysm diameter & 0.97 & .43 \\
Aneurysm neck & 1.04 & .73 \\
Associated thrombus & 1.60 & .60 \\
\hline
\end{tabular}

${ }^{a}$ Mode of presentation was analyzed as a global model categorizing presentations as cranial nerve deficit, headache, incidental, neurologic deficit (noncranial nerve), recanalization of previously treated aneurysm, or other. Global model not significant; individual predictors also not significant (odds ratios and $P$ values not shown).

\section{Clinical Outcomes and Complications}

At most recent follow-up, $89.3 \%$ of patients are well, with either no deficit or a stable or improved pre-existing deficit (84 of 94 patients). Four patients (4.3\%) have new, persistent neurologic deficits, all of which are improving at last follow-up. Overall mortality in the series is $6.4 \%$ ( 6 cases), as summarized in the On-line Table. In univariate analyses (Table 3), only aneurysm location in the posterior circulation was associated with increased odds of mortality. There was a trend toward increased mortality among females. Of the 10 patients presenting with visual loss, 1 died (Patient 4, On-line Table) and 9 were available for follow-up. Of these, 4 remained stable, 4 have significantly improved vision, and 1 had complete resolution of visual deficit. Of the 5 patients presenting with a neurologic deficit, 1 died (Patient 6, On-line Table), 2 are stable, 1 has improved, and 1 deficit has resolved. The outcomes for oculomotor cranial neuropathies are reviewed with the cavernous aneurysms below.

Hemorrhage from the treated aneurysm was documented in 3 cases $(3.2 \%)$. One case (previously reported ${ }^{5}$ ) resulted in brain stem hemorrhage and death (Patient 1, On-line Table). In another case, there was a trace of subarachnoid blood noted on imaging, but death was attributed to acute aneurysm thrombosis and hydrocephalus (Patient 5, On-line Table). The third case occurred over 1 year after aneurysm treatment. This patient had a complex previously ruptured and coiled carotid termination aneurysm. The aneurysm continued to fill after treatment with a single Pipeline stent into the middle cerebral artery. An attempt was made to coil the residual aneurysm and anterior cerebral artery outlet from the contralateral side. This failed and the patient declined further treatment. She then presented with hemorrhage from the index aneurysm. This was managed with surgical occlusion of the A1 segment of the anterior cerebral artery, followed by placement of second Pipeline stent.

Distal ipsilateral parenchymal hemorrhage occurred in 4 cases (4.3\%). One patient died (Patient 2, On-line Table). One patient remained well without intervention. Two patients have persistent but improving neurologic deficits, 1 required surgical evacuation of the hematoma, while the other was managed conservatively. In addition to the case mentioned above (Patient 5, On-line Table), 1 other patient developed acute hydrocephalus secondary to thrombosis of a vertebrobasilar junction aneurysm. This was successfully managed with endoscopic third ventriculostomy.

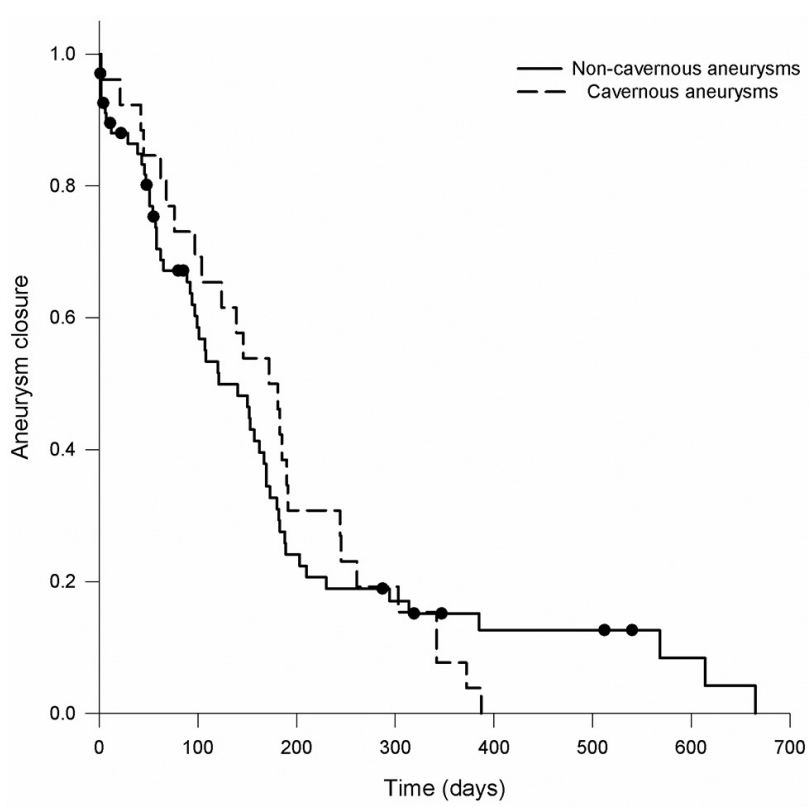

FIG 3. Kaplan-Meier survival plot comparing the rate of aneurysm closure for cavernous segment aneurysms versus other locations. Shaded circles indicate censored observations.

\section{Cavernous Segment Aneurysms}

Cavernous segment aneurysms represent a unique subset of the overall cohort. A total of 26 cavernous segment aneurysms were treated, $70 \%$ presenting with cranial neuropathy. This subset tended to be larger in size (mean diameter $21.4 \mathrm{~mm}$ versus 17.6 $\mathrm{mm}, P=.05)$. There was also a trend toward increased partial thrombosis of the aneurysm fundus, observed in $38 \%$ of cavernous aneurysms versus $20 \%$ for all other aneurysms $(P=.07)$. Aneurysm closure is probably superior in this category. Although survival analysis does not indicate any increased rate of closure over time ( $P=.90$; Fig 3$)$, the proportion of aneurysms going on to complete or near-complete occlusion is higher. All cavernous aneurysms (100\%) achieved complete or near-complete occlusion through follow-up compared with $78 \%$ of noncavernous carotid locations $(P=.005)$.

Serious neurologic complications did not occur in this cohort, with no incidence of distal ICH or aneurysmal hemorrhage. There were no deaths ( $0 \%$ mortality) in this subset, compared with 6 deaths in noncavernous locations $(8.5 \%)$. New or worsened cranial nerve deficits occurred in 3 patients $(11.5 \%)$ after treatment with the PED. In 2 cases, the cranial nerve deficit has resolved, whereas 1 of the patients has persistent though improved diplopia. There were 2 periprocedural minor embolic strokes related to tortuous access to the aneurysm. In both cases, the associated neurologic deficit-mild hemiparesis—resolved in follow-up. For patients presenting with cranial nerve deficit (18/26 cavernous aneurysms), 11 have complete resolution (61\%), with subtotal improvement noted in an additional 2 cases (11\%) and no significant change in the remaining 5 cases $(28 \%)$.

\section{DISCUSSION}

Review of the initial Canadian experience with the PED demonstrates effective angiographic closure of the treated aneurysms, with $84.2 \%$ complete or near-complete occlusion through an av- 
erage of 1.25 years follow-up. Survival analysis indicates progressive aneurysm closure over time. These results are comparable with other studies in the literature reporting angiographic aneurysm occlusion rates in between $74 \%$ and $94 \%$ of cases. ${ }^{2,3,6-11}$ In the current study, multivariate analysis suggested female sex and previous aneurysm treatment as predictors of persistent aneurysm filling. Many studies do not comment on predictors of aneurysm occlusion after flow-diverting stent placement. Although small numbers precluded statistical analysis, McAuliffe et $\mathrm{al}^{8}$ identified fusiform aneurysms, smaller aneurysm size, and previous aneurysm treatment as predictors of persistent angiographic filling in follow-up. Further study is required to better predict successful aneurysm occlusion with flow-diverting stents. The most favorable results in this initial Canadian experience came from the subset of cavernous segment aneurysms, with all cavernous aneurysms closed by 1 year. There were no deaths and significant morbidity was uncommon. Most patients presenting with cranial nerve deficits were resolved or improved in follow-up. Flow-diverting stents may represent an important treatment option for patients with large cavernous aneurysms; however, one must consider that proximal occlusion is a safe and effective treatment in appropriately selected patients. ${ }^{12}$

There were 2 general sources of morbidity after PED treatment of the aneurysms in this series: failure to alter the natural history or complications related to placement of the device. The natural history of unruptured aneurysms has never been clearly defined. Nevertheless, most studies have shown that large aneurysms, such as those treated in this study, have a high risk of rupture and neurologic morbidity due to progressive growth. ${ }^{13}$ In many cases, the PED appears to avert this natural history by leading to aneurysm occlusion and even regression of the aneurysm mass. However, there are important exceptions. Despite successful PED placement, aneurysm rupture occurred in 3.2\% of cases in this series. One of the cases occurred over 1 year after the initial treatment in an aneurysm that demonstrated persistent filling despite the successful PED placement. Before presenting with rehemorrhage, the patient had declined further intervention. The other cases bled relatively early after partial thrombosis of the treated aneurysms. This pattern is similar to other reports in the literature and has been postulated by others to relate to thrombus-mediated weakening of the aneurysm wall. ${ }^{5,14}$ In this manner, it is possible that the flow diversion may actually increase the risk of rupture after treatment. The true incidence of aneurysm rupture after flow-diverting stent placement remains unknown. Kulcsár et $\mathrm{al}^{14}$ reported a series of 13 similar cases pooled from multiple institutions; however, the appropriate denominator could not be established. In other series, this delayed aneurysm rupture is a relatively rare occurrence, observed in $0 \%,{ }^{3,9} 1 \%,{ }^{6}$ and $1.4 \%{ }^{6}$ of cases depending on the report. In the absence of aneurysm rupture, failure to alter the natural history can manifest as progressive aneurysm growth. In the Canadian experience, there were 3 cases of progressive aneurysm enlargement despite the flow-diverting stent. Importantly, this progression in size corresponded to adverse neurologic outcomes.

The second source of morbidity after placement of a flowdiverting stent is procedure-related complication. As with any endovascular procedure, periprocedural stroke is an important potential risk. In our series, symptomatic periprocedural ischemic stroke was relatively rare, occurring in $4 \%$ of cases, with persistent, but improving, deficit in $2 \%$. This study did not consider new silent ischemic events, as postprocedural MR imaging scans were not available for all patients. Embolic events may occur at increased frequency, given the size of the delivery devices and the complexity of cranial vascular access seen in typical flow-diversion cases. On the other hand, dual antiplatelet therapy has been associated with a reduction in thromboembolic events in series using conventional coiling ${ }^{15,16}$ and with stent-assisted coiling techniques. ${ }^{17}$ Distal ipsilateral hemorrhage has emerged as a more specific complication related to flow diversion. In our Canadian experience, distal hemorrhage occurred in $4 \%$ of cases, resulting in significant morbidity and mortality. The incidence of these hemorrhages varies in the literature, with no occurrences in most series. ${ }^{3,6,8,9,11}$ Fischer et $\mathrm{al}^{2}$ reported 3 cases in their experience (101 cases) with the PED. The putative mechanism is unknown, but hemorrhagic conversion of ischemic embolic events, due to antiplatelet medication, combined with altered pressure dynamics, due to redirected flow, have been postulated. ${ }^{18}$ Although the phenomenon appears to be relatively specific to flow diversion, it has been described with conventional stent-assisted coiling procedures. ${ }^{19}$ Finally, aneurysms may undergo an increase in size after treatment due to acute thrombosis and perianeurysmal edema. In cavernous aneurysms, this manifested as new cranial neuropathy. Most of these resolved over time; however, certain patients had persistent deficits. This acute increase in aneurysm mass effect also resulted in hydrocephalus, necessitating a surgical intervention (third ventriculostomy). If hydrocephalus is a potential concern on the basis of the preoperative imaging, prophylactic intervention before initiation of antiplatelet medication may be beneficial.

Allowing for overlap of specific complications, the overall morbidity and mortality rate in this series is $10.7 \%$ (6.3\% mortality, $4.4 \%$ morbidity). The observed morbidity in this series must be considered in the context of the existing therapeutic alternatives. Effective surgical treatment of large aneurysms can frequently be achieved, with acceptable morbidity and mortality. More elaborate surgical procedures, such as high-flow bypass and circulatory arrest, have elevated procedural risk. In a recent report from a high-volume center, surgery for giant aneurysms using bypass and proximal occlusion techniques has a reported mortality of $13 \%$ and permanent neurologic morbidity of $9 \%{ }^{20}$ Similarly, in a series of 105 patients with hypothermic circulatory arrest at the Barrow Neurological Institute, the combined procedure-related morbidity and mortality was $32 \% .{ }^{21}$ Conventional endovascular approaches such as coiling with balloon or stent assistance may have relatively lower procedural risk; however, these interventions have been shown to have diminished efficacy for large, complex aneurysms leading to an increased risk of recurrence and retreatment. In a systematic review of giant aneurysms treated with conventional endovascular approaches, the complete occlusion rate was $57 \%$, with recanalization in $27 \% .^{22}$ The rates of morbidity and mortality were $17 \%$ and $8 \%$, respectively. ${ }^{22}$

Given that flow-diverting stents are a relatively recent innovation, their performance through long-term follow-up remains to 
be evaluated. Imaging protocols varied significantly between and within the participating centers. In our experience, CTA is preferred over MRA in cases where there are not additional coils or clips causing artifacts. Contrast-enhanced CTA and MRA appear to be sufficient to evaluate for residual aneurysm filling as well as the residual aneurysm mass. Neither technique is suited to evaluating in-stent stenosis. DSA can be used at 6 months to screen for in-stent stenosis, thereby allowing a decision to be made regarding continuation of dual antiplatelet therapy. The optimal use and need for DSA in long-term follow-up requires further analysis.

Significant in-stent stenosis is relatively rare, occurring in 2 cases in this series, with both patients remaining relatively stable or improving over time. Of greater concern are our 2 cases of delayed vessel occlusion. Fortunately, neither patient suffered lasting neurologic consequences because of aneurysm locations in the anterior circulation with intact collateral circulation. Fiorella et $\mathrm{al}^{23}$ reported a case with delayed stent thrombosis in the posterior circulation, with significant morbidity. The timeframe for most studies in the literature precludes effective analysis of this phenomenon. As such, the relative uncertainty in long-term performance should be factored into clinical decision-making surrounding potential flow-diversion interventions.

This study suffers from a number of limitations, given its retrospective and nonrandomized nature. Outcomes were self-reported by the treating interventionalist, introducing potential measurement bias. Standardized outcome measures such as angiographic grading scales and clinical outcome scales were not uniformly applied and were not available for complete analysis. Due to the proctoring requirements, case selection and stent deployment were subject to expert supervision, limiting generalizability of the data. This study was not, however, intended to provide definitive answers. Rather, this study reports a nascent experience with a novel technology used to treat challenging intracranial aneurysms. The purpose of this review is to highlight areas for further research and study. Focused randomized trials are needed determine the relative efficacy of flow-diverting stents compared with standard aneurysm treatment. Further data are needed on long-term outcomes as well as predictors of aneurysm closure and procedural complications. Finally, even if flow-diverting stents can be established as a safe and effective treatment for intracranial aneurysms, it must be determined whether these expensive devices are cost-effective for health care systems. Of note, the cost of a single PED approaches 3-4 times that of a conventional intracranial stent. As a result, a case using a single PED may have a similar cost to stent-assisted coiling; however, the costs rise exponentially with multiple PED use. Whether cost savings are realized in prevention of recurrence or complications requires further analysis.

\section{CONCLUSIONS}

Flow-diverting stents such as the PED represent an important tool in the treatment of complex intracranial aneurysms. These stents have the potential to achieve dramatic results in difficult aneurysms, but complications and treatment failure can occur. Our results suggest that enthusiasm for this technology should be tempered by a careful consideration of the efficacy and morbidity of these devices compared with more standard treatment alterna- tives. Ongoing analysis of flow-diverting stents for radiographic and clinical performance is required.

Disclosures: Michael Kelly—RELATED: Support for Travel to Meetings for the Study or Other Purposes: Covidien-ev3, Comments: Pipeline proctors course in Anakara, Turkey, April 2011, and Pipeline Users Meeting, Calgary, AB, January 2012-reimbursement for travel expenses only; OTHER RELATIONSHIPS: The 2 meetings were about the Pipeline embolization device, which is one of the devices reported in the manuscript. Thomas R. Marotta—RELATED: Consulting Fee or Honorarium: Proctoring for PED from ev3, Covidien; Support for Travel to Meetings for the Study or Other Purposes: Proctoring for PED from ev3, Covidien; UNRELATED: Consultancy: Proctoring for PED from ev3, Covidien; Patents (planned, pending, or issued): EVASC medical: eclips - endovascular clip system. Drs. Marotta, Kelly, and O'Kelly have served as proctors for ev3 Endovascular on Pipeline deployment cases.

\section{REFERENCES}

1. Bendok BR, Parkinson RJ, Hage ZA, et al. The effect of vascular reconstruction device-assisted coiling on packing density, effective neck coverage, and angiographic outcome: an in vitro study. $\mathrm{Neu}$ rosurgery 2007;61:835-40; discussion 840-41

2. Fischer S, Vajda Z, Aguilar Perez M, et al. Pipeline embolization device (PED) for neurovascular reconstruction: initial experience in the treatment of 101 intracranial aneurysms and dissections. Neuroradiology 2012;54:369-82

3. Lylyk P, Miranda C, Ceratto R, et al. Curative endovascular reconstruction of cerebral aneurysms with the Pipeline embolization device: the Buenos Aires experience. Neurosurgery 2009;64:632-42; discussion 642-43; quiz N6

4. Fuller E, Marotta TR, Chen JM, et al. Middle ear aneurysm treated with an innovative, vessel-preserving, aneurysm-occluding stent. Laryngoscope 2010;120:796-99

5. Chow M, McDougall C, O’Kelly C, et al. Delayed spontaneous rupture of a posterior inferior cerebellar artery aneurysm following treatment with flow diversion: a clinicopathologic study. AJNR Am J Neuroradiol 2012;33:E46-51

6. Byrne JV, Beltechi R, Yarnold JA, et al. Early experience in the treatment of intra-cranial aneurysms by endovascular flow diversion: a multicentre prospective study. PLoS One 2010;5

7. Lubicz B, Collignon L, Raphaeli G, et al. Flow-diverter stent for the endovascular treatment of intracranial aneurysms: a prospective study in 29 patients with 34 aneurysms. Stroke 2010;41:2247-53

8. McAuliffe W, Wycoco V, Rice $\mathrm{H}$, et al. Immediate and midterm results following treatment of unruptured intracranial aneurysms with the Pipeline embolization device. AJNR Am J Neuroradiol 2012;33:164-70

9. Nelson PK, Lylyk P, Szikora I, et al. The Pipeline embolization device for the intracranial treatment of aneurysms trial. AJNR Am J Neuroradiol 2011;32:34-40

10. Szikora I, Berentei Z, Kulcsar Z, et al. Treatment of intracranial aneurysms by functional reconstruction of the parent artery: the Budapest experience with the Pipeline embolization device. AJNR Am J Neuroradiol 2010;31:1139-47

11. Wagner A, Cortsen M, Hauerberg J, et al. Treatment of intracranial aneurysms. Reconstruction of the parent artery with flow-diverting (Silk) stent. Neuroradiology 2012;54:709-18

12. Niiro M, Shimozuru T, Nakamura K, et al. Long-term follow-up study of patients with cavernous sinus aneurysm treated by proximal occlusion. Neurol Med Chir (Tokyo) 2000;40:88-96; discussion 96-97

13. Wiebers DO, Whisnant JP, Huston J 3rd, et al. Unruptured intracranial aneurysms: natural history, clinical outcome, and risks of surgical and endovascular treatment. Lancet 2003;362:103-10

14. Kulcsár Z, Wetzel SG, Augsburger L, et al. Effect of flow diversion treatment on very small ruptured aneurysms. Neurosurgery 2010;67:789-93 
15. Hwang G, Jung C, Park SQ, et al. Thromboembolic complications of elective coil embolization of unruptured aneurysms: the effect of oral antiplatelet preparation on periprocedural thromboembolic complication. Neurosurgery 2010;67:743-48; discussion 748

16. Yamada NK, Cross DT 3rd, Pilgram TK, et al. Effect of antiplatelet therapy on thromboembolic complications of elective coil embolization of cerebral aneurysms. AJNR Am J Neuroradiol 2007;28:1778-82

17. Brooks NP, Turk AS, Niemann DB, et al. Frequency of thromboembolic events associated with endovascular aneurysm treatment: retrospective case series. J Neurosurg 2008;108:1095-100

18. Cruz JP, Chow MC, O'Kelly CJ, et al. Delayed ipsilateral parenchymal hemorrhage following flow diversion for the treatment of anterior circulation aneurysms. AJNR Am J Neuroradiol 2012;33:603-08

19. Kim DJ, Suh SH, Kim BM, et al. Hemorrhagic complications related to the stent-remodeled coil embolization of intracranial aneurysms. Neurosurgery 2010;67:73-78; discussion 78-79

20. Sughrue ME, Saloner D, Rayz VL, et al. Giant intracranial aneurysms: evolution of management in a contemporary surgical series. Neurosurgery 2011;69:1261-70; discussion 1270-71

21. Ponce FA, Spetzler RF, Han PP, et al. Cardiac standstill for cerebral aneurysms in 103 patients: an update on the experience at the Barrow Neurological Institute. Clinical article. J Neurosurg 2011;114:877-84

22. Parkinson RJ, Eddleman CS, Batjer HH, et al. Giant intracranial aneurysms: endovascular challenges. Neurosurgery 2006;59:S10312; discussion $\mathrm{S} 3-13$

23. Fiorella D, Hsu D, Woo HH, et al. Very late thrombosis of a Pipeline embolization device construct: case report. Neurosurgery 2010;67: E313-14; discussion E314 